\title{
Proportional QoS over WDM Networks: Blocking probability
}

\author{
Yang Chen \\ Electrical and Electronic Engineering \\ Department \\ Hong Kong University of \\ Science and Technology
}

\author{
Mounir Hamdi \\ Computer Science Department \\ Hong Kong University of \\ Science and Technology \\ hamdi@cs.ust.hk
}

\author{
Danny H. K. Tsang \\ Electrical and Electronic Engineering \\ Department \\ Hong Kong University of \\ Science and Technology
}

\begin{abstract}
The provision of scalable quality-of-service (QoS) guarantees on wavelength-division- multiplexing (WDM) networks is an important and challenging issue for the next generation Internet. One of the important performance metrics in a QoS-capable WDM network is the call blocking probability. Recently, a proportional differentiation model has been proposed as an effective method for scalable differentiated services provision. And this model provides the network operators the ability of quantitatively adjusting the quality differentiation between service classes. In this paper, we introduce this model into WDM networks with the aim of providing proportionally differentiated blocking probability to various traffc classes. An intentional blocking algorithm is proposed to implement this model at the wavelength level. In order to solve the link utilization degradation in this algorithm, we propose another intentional termination algorithm. Since the performance requirement from the network operator might be various, a hybrid algorithm is also given as a balance between the above two. These three algorithms are also suitable to TDM over WDM, where one connection only take part of the transmission capacity of one wavelength. Extensive simulation results demonstrate that our algorithms provide accurate and controllable differentiation on blocking probability between various traffc classes even in a bursty traf£c situation. Infeasibility problem in proportional blocking probability provision is discussed at the end of this paper.
\end{abstract}

\section{Introduction}

Wavelength Division Multiplexing (WDM) is the use of multiple wavelengths to transmit different data streams simultaneously. WDM is attractive since the same fber can be used for different channels, saving costs on fber and installation [1]. With the recent advances in optical device and technology, an optical £ber with more than one hundred wavelengths has been demonstrated. As a result, WDM network has the potential of delivering an aggregated throughput in the order of Terabits per second, which makes WDM network to be the ideal solution for the ever-growing demand for more bandwidth. Naturally, it has been the subject of extensive research and development by both academia and industry.

Currently, most WDM backbone networks employ circuit switching for the exchange of aggregated traffc between nodes because it is much easier to design a circuitswitched router than a packet-switched router using optical technology. These circuits are established over lightpaths from source to destination. These lightpaths could use a single wavelength from source to destination in which case routers do not have wavelength-conversion capability. On the other hand, a lightpath can be established over multiple wavelengths where the routers would have wavelength conversion capability. Since the link utilization will be poor if sessions require only a fraction of a wavelength's capacity, Time Division Multiplexing (TDM) in WDM network is proposed recently [6]. As shown in Figure 1, a session request is assigned one or more time slots forming an optical connection, for the duration of the session. In either case, one key measure for the performance of these WDM networks is the blocking probability from source to destination. That is, a connection (Mow-level, aggregated-level, or virtual-circuit-level) cannot be established from a source node to a destination node because of lack of bandwidth. The bandwidth can be measured in terms of discrete number of wavelength or as a portion of a wavelength.

A lot of the research works on blocking probability in WDM networks are centered on two issues. The frst is that given a certain wavelength routing and assignment (RWA) algorithm, how to determine the blocking probability for a given network (with given topology and number of wavelengths and routers capabilities). The analysis of call blocking probabilities under static ( $\mathfrak{e x e d}$ or alternate) routing with random wavelength allocation and with or without wavelength converters has been studied in $[2,3]$. The sec- 


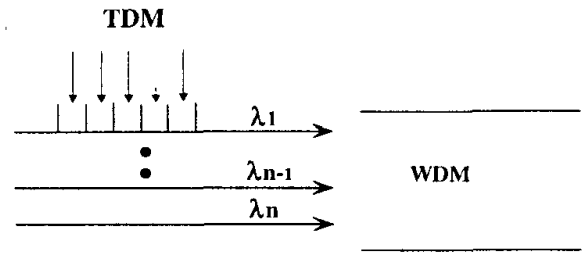

Figure 1. Time Division Multiplexing on a WDM link

ond issue is how to design a RWA algorithm for a given WDM network that minimizes the blocking probability [5]. Current work on TDM over WDM focuses on maximizing network throughput. But we believe blocking probability of sessions is also an important performance parameter.

Unfortunately, most of the research efforts in this area assume that the entire connection establishment is for a single class of traffc. In a recent research [7], the WDM blocking probability analysis has been extended to multi-class services. However, the difference between services only exist at the call arrival rates and the call holding times. In this paper, we will introduce service quality differentiation into WDM networks.

The rest of the paper is organized as follows. Section 2 introduces previous works on QoS provision in circuit switched network and today's WDM networks. Different QoS models with particular attention given to proportional service model are described in Section 3. In Section 4, we give algorithms that achieve proportional blocking probability in WDM networks and TDM/WDM network. Section 5 quantifes the effectiveness of our algorithms through extensive simulation results. We discuss the infeasibility problem in the proportional QoS model and possible feasibility testing method in Section 6. Finally, this paper is concluded in Section 7.

\section{Previous work}

Average delay for traf£c is chosen as the QoS metric in [8]. In this QoS framework, three types of paths: (i) Dedicated lightpath; (ii) Shared lightpath; and (iii) Multihop path are provided by the underlay optical WDM networks. Traffc using Dedicated Lightpath will experience delay caused only by propagation and O/E, E/O conversion at the access node. Traffc using Shared lightpath will possibly experience more delay in buffer since the lightpaths are shared by multiple traffc dows. Since Multi-hop path covers multiple lightpaths, delay for the $\mathrm{O} / \mathrm{E}, \mathrm{E} / \mathrm{O}$ conversion and buffering at the junction between two lightpath will be added to the total delay for a traffc transmitted over this kind of path.
In order to provide differentiation on blocking probability in telecommunication networks, the usage of trunk reservation is discussed in [9]. In general, a connection request belonging to service class $i$ is accepted if the free capacity after this connection's admission is at least equal to the sum of the trunk reservation of the higher priority class. Formally, suppose the total capacity of the link is $C$; let $r_{i}$ be the amount of capacity already occupied by class $i$ 's connection; $t_{i}$ is the trunk reservation for class $i$; a new connection request of class $i$ have a capacity request of $r$ is accepted when ${ }^{1}$ :

$$
\sum_{j=1}^{N} r_{j}+r+\sum_{j<i} t_{j} \leq C
$$

A small amount of trunk reservation can give obvious priority of one service class over others. Here is an example: suppose we have a WDM link with 64 wavelengths; there are 4 service classes; the trunk reservation for class 0 to 3 are all one wavelength.

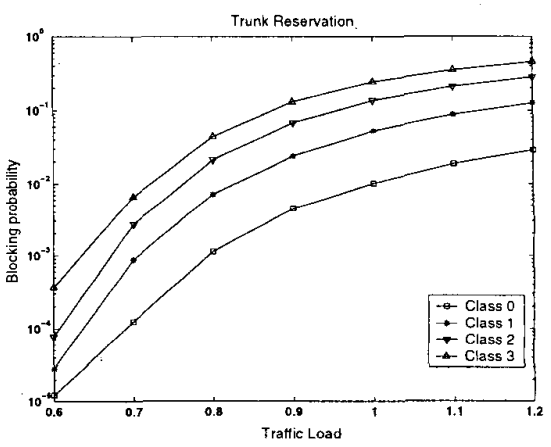

Figure 2. The effect of trunk reservation

In Figure 2, we can $£$ nd that trunk reservation can give good differentiation on the call blocking probability among different service classes. But the differentiation are not controllable to the network operators. Then it is not suitable for implementation of proportional QoS model introduced in the following section.

\section{Proportional QoS}

Wide diversity on the service requirements of users and applications on today's Internet makes the best effort service model inadequate. Hence, there is a great demand for the Internet to be extended with service differentiation.

\footnotetext{
${ }^{1}$ In the whole paper, we assume class $i$ has higher priority than class $j$ if $i>j$.
} 


\subsection{Previous QoS models}

The frst approach proposed to replace the best effort model is Integrated Services (Intserv) [10]. Using resource reservation and admission control (through protocols like RSVP), application's request for a certain level of performance can be guaranteed. But per-dow state information should be kept inside each router along the way in order to fulfll the service requirement of each xow. As a result, this approach encounters a scalability problem in its deployment. In order to overcome the scalability problem of Intserv, a relatively new approach: Differentiated Service (Diffserv) [11] has been proposed. Instead of providing endto-end per- $x_{0}$ ow performance guarantees, Diffserv provides local (per hop) service differentiation for aggregated traf$f c$ with the same QoS requirement (per class). This goal is achieved by defning packets' Per-Hop-Behavior (PHB) at each router. No state information about each tow is kept in the core of the network due to its per-class QoS model. In particular, two models of Diffserv have been identifed: Absolute Service Differentiation and Relative Service Differentiation. The latter is receiving more attention because of its simplicity and its ability to be deployed incrementally [12]. Recently, this relative QoS model has been further refned using a proportional differentiation model, which provides the network operators with quantitative QoS differentiation between service classes $[12,13]$.

\subsection{Proportional differentiation model}

In a relative QoS model, we can only guarantee that the traffc from a higher priority class will receive no worse local service. As an improvement, in the proportional differentiation model, we can quantitatively adjust the service differentiation of a particular QoS metric to be proportional to the differentiation factors that a network service provider sets beforehand. If $q_{i}$ is the QoS metric of interest and $s_{i}$ is the differentiation factors for class $i$, using the proportional differentiation model, we should have:

$$
\frac{q_{i}}{q_{j}}=\frac{s_{i}}{s_{j}} \quad(i, j=1 \ldots N)
$$

for all pairs of service classes. For example, in a packet network, assume that $q_{1}, q_{2}$ are the packet loss probability for class 1 and 2 respectively. If $s_{1}$ is 1 while $s_{2}$ is 2 , then the packet loss probability of class 2 should be twice that of the packet loss probability of class 1 .

It is desirable that the proportional differentiation model holds over not only long time scales, but also short time periods. The reason is that the long term average is not quite meaningful when the traffc is bursty. Then the proportional differentiation equation (1) should hold within a short time period $\tau$, which is called monitoring timescale in [12] :

$$
\frac{\overline{q_{i}}(t, t+\tau)}{\overline{q_{j}}(t, t+\tau)}=\frac{s_{i}}{s_{j}}
$$

where $\bar{q}_{i}(t, t+\tau)$ is the average QoS metric in the time period $\tau$. This service model is general enough in that the quality differentiation between traffc classes can be defned as a function of various QoS parameters. From equation (2), we can see that the proportional QoS model is controllable: by changing the quality differentiation factors, the QoS differentiation between certain service classes can be adjusted. In addition, this model is also predictable: if $\tau$ is small enough, the traffc in a higher priority class will consistently receive better service than a lower priority class independent of the load situation.

In the previous section, we learn that using trunk reservation can provide blocking probability differentiation between service classes. In the trunk reservation, the low priority call arrival will be blocked if the remaining capacity is less than the trunk reservation. This will lead to higher blocking probability for low priority call arrival. And the reserved capacity will reduce the probability that a high priority call does not fnd enough capacity. Enlarging trunk reservation value will give better differentiation. But we can not have a proportional result. Some modifcations must be done to the previous scheme. We introduce the intentional blocking algorithm frst in the following section, which decides a call's admission or blocking with the on-line measurement of the blocking probability of each class.

\section{Algorithms}

\subsection{Intentional Blocking}

In stead of setting a £xed trunk reservation for each service class, which leads to uncontrollable blocking probability, we do the admission control on the quantitative measurement. When the blocking probabilities of different classes deviate from the proportional equation (2), we block the call arrival even there has free capacity available on the link.
Defne:
block $_{i}$
arrival $_{i}$
$s_{i}$
ERROR
$\operatorname{prob}_{i}=\frac{\text { block }_{i}}{\text { arrival }_{i} * s_{i}}$
number of call blocked for class $i$; number of call arrived for class $i$; proportional factors set for class $i$; a parameter controls the accuracy of proportional relations;
online weighted blocking probability
measurement for class $i$; 
1. Set two counters recording block $k_{i}$ and arrival $_{i}$ to be 0 for each class $i$;

2. When a call $c_{i}$ of class $i$ arrives, if ( $c_{i}$ can not fnd free wavelength or capacity) $c_{i}$ is blocked, block $_{i}+1$, arrival $i+1$ else

\{

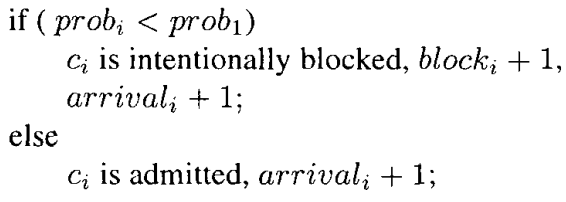

3. Update $p r o b_{i}$, compute difference between $\operatorname{prob}_{1}$ and $\operatorname{prob}_{j}(j=2 \ldots N)$.

if (all the differences are less than ERROR)

a "matching" happens, go to step 1 ;

else go to step 2.

In the above algorithm, we just use the blocking probability of class 0 as the reference for the proportional condition testing. Thus, we can reduce the computing effort from $O\left(N^{2}\right)$ (in equation (2)) to $O(N)$. In addition, by resetting counter from time to time, we can avoid the counter overaowing problem while using the most recent information in the measurement, which is preferable when the traffc is bursty. Since we do not admit call arrival when there is free capacity, we can expect link utilization degradation compared with complete sharing. We are able to increase the link utilization using the following intentional termination algorithm.

\subsection{Intentional Termination}

The terms defnitions are the same as intentional blocking algorithm.

\section{Algorithm:}

1. Set two counters recording block b $_{i}$ and arrival $_{i}$ to be 0 for each class $i$;

2. When a call $c_{i}$ of class $i$ arrives, if ( $c_{i}$ fnd free wavelength or capacity) $c_{i}$ is admitted, arrival $_{i}+1$; else

\{

if $\left(p r o b_{i}<p r o b_{1}\right)$ $c_{i}$ is blocked, block $_{i}+1$, arrival $i+1 ;$ else

\{

if there exists a connection $c_{j}$ belongs to class $j, j \neq i$ and $\operatorname{prob}_{j}=\max \left\{\operatorname{prob}_{k} \mid k=2 \ldots N\right\}$

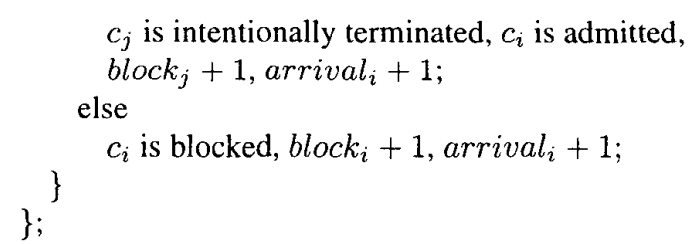

3. Update $p r o b_{i}$, compute difference between $p r o b_{1}$ and $\operatorname{prob}_{k}(k=2 \ldots N)$.

if (all the differences are less than $E R R O R$ ) a "matching" happens, go to step 1;

else go to step 2.

In this algorithm, we can expect high link utilization as good as complete sharing. But we will terminate some connections before their release time. This might start the complex restoration or protection process in the some optical networks. As a balance between the intentional blocking and termination, the hybrid algorithm is proposed.

\subsection{Hybrid Algorithm}

This algorithm actually is a combination of the above two algorithms with only one additional parameter $p, 0 \leq$ $p \leq 1$.

When a call arrival fnds there is free capacity, it will have a probability $p$ to initiate the intentional blocking probability. Meanwhile, a call arrival Ends there is no free capacity, it will have a probability $1-p$ to initiate the intentional termination algorithm. The information recorded in the counters will be used by those two algorithms. By changing the value of $p$, we can have a smooth transition from intentional blocking to termination algorithm. Simulation result on the link utilization comparison among these three algorithms will be given later.

\subsection{TDM over WDM}

With little modifcation to the above three algorithms: change the capacity assign level from one wavelength to one time slot in the frame on a wavelength. we can easily provide proportional blocking probability to the sessions asking for one time slot in the frame.

\section{Simulation Results}

Since the TDM over WDM simulation is not different from WDM link except the volume of assigned capacity, we only give simulation results on WDM link in this paper.

We assume a link with 64 wavelengths on it. There are four classes of call arrivals. One call need one wavelength for connection setup. Each class consists $25 \%$ of the total amount of traffc. We set the proportional factors to be: 
$s_{0}=1 ; s_{1}=2 ; s_{2}=4 ; s_{3}=8 ;$. The ERROR is: 0.0001 . We use Poisson traffc source this time. Since all the three algorithms give similar blocking probability differentiation, we only show the performance of intentional blocking in Figure 3. The link utilization comparison of these three algorithms is shown in Figure 4.

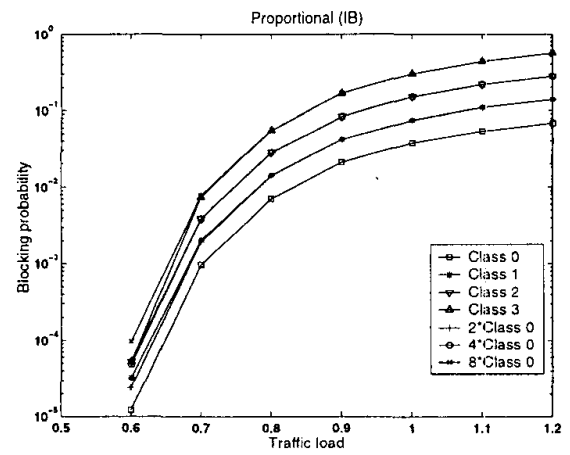

Figure 3. Proportional blocking probability

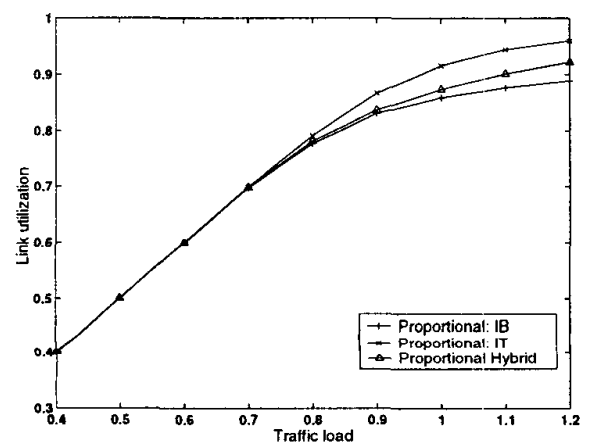

Figure 4. Link utilization comparison

We can fnd some deviation when the traf£c load is light. The explanation is when traffc load is light, the blocking probability is so small that it is comparable to the value of $E R R O R$, which is 0.0001 in this set of simulation. Then in each "matching" process, the proportional relationship is not strictly kept. This leads to the deviation in the proportional QoS provision. We can decrease the value of ERROR to make the proportional QoS provision more accurate when the traffc load is quite low. This will cause the longer time between counter resetting.

In order to test our algorithms when the traffe is bursty, we change the poisson traffc source in the above simulation to a bursty traf $\mathcal{L} \mathrm{c}$ source. The traffc source have 5 states, in state 0 , there call arrival rate is 0 ; in state 1 , the arrival rate is $r$; as the traffc source moves to a higher neighbor state, the arrival rate will increase $r$. The transition time period between states following a exponential distribution.
The value of $E R R O R$ is set to be 0.0005 . The performance of each algorithms are shown in Figure 5

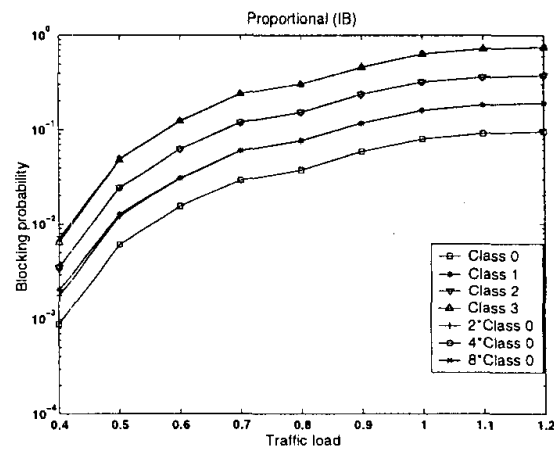

Figure 5. Proportional blocking probability: Bursty traf£c

We now test our algorithm for the load distribution auctuation. The simulation scenario is: a WDM link with 160 wavelengths; 3 type of call arrivals; $s_{0}=1 ; s_{1}=2 ; s_{2}=$ 4 ; ; the total traf£c load is kept as $95 \%$; at the 260,001 call arrival, the percentage changes from Traf£c 1: 20\%; Traffc 2:30\%; Traffc 3: $50 \%$ to be: $50 \%, 30 \%, 20 \%$ respectively; $E R R O R=0.0005$; Then the call blocking probability for

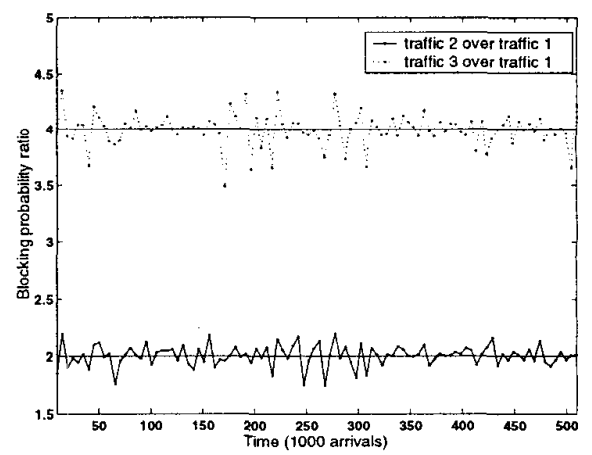

Figure 6. Proportional differentiation in trafEc percentage change (measured every 5000 arrivals)

each traffc class is computed every 5000 call arrivals. The blocking probability ratio is shown in Figure 6. We can see that the blocking probabilities increase to some extent after the percentage change. But the blocking probability ratios between different traf£c class are not affected by the percentage change. This simulation result further show that our algorithms not only $£ t$ equation (2) well, but also consistent to equation (3). 


\section{Feasibility}

Here is one example showing that we may not achieve the proportional blocking probabilities using our algorithms. Suppose a link with 8 wavelengths; 4 service classes each consists $25 \%$ of the total traffc; $s_{0}=1 ; s_{1}=2 ; s_{2}=$ $4 ; s_{3}=8$;. We using intentional termination in this simulation. As we can fnd in Figure 7, when the load is high,

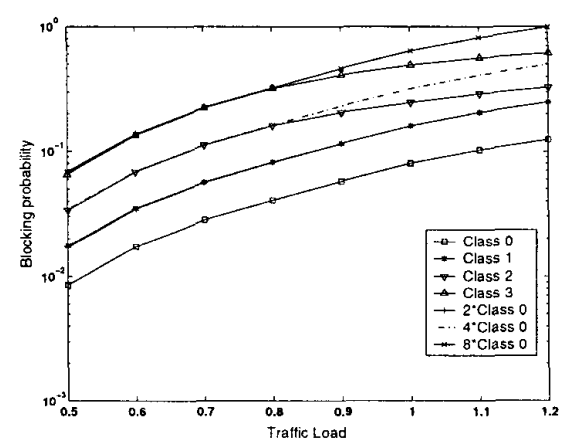

Figure 7. Infeasible proportional blocking probability)

we can not achieve a proportional blocking probability differentiation. The explanation is when the load is high, the blocking probability for class 2 and 3 are high, the probability that a class 0 ends a connection of class 2 or 3 occupying one of the 8 wavelengths is not negligible. When a class 0 call need to terminate low priority connection, it will not be able to fnd such a connection from time to time. So in this load situation and with current proportional factors, we can not achieve corresponding proportional blocking probability. A scheme for testing the feasibility of a set of proportional factors under particular load situation will be reported in future work.

\section{Conclusions}

In this paper, we introduce the intentional blocking/termination algorithms, which give a proportional call blocking probability on WDM links. From simulation results, we fnd our algorithms provide accurate proportional differentiation even in a small time scale and independent from traffc load shifting or percentage change, which is especially important for bursty traffc handling. The infeasibility problem in proportional blocking probability provision is discussed. Future work including proportional QoS provision within general mesh network situation and guide line for choosing feasible proportional factors will be reported in up-coming publications.

\section{References}

[1] R. Ramaswami and K. Sivarajan. "Optical networks: a practical perspective," Morgan-Kufmann Publisher, 1998.

[2] A. Birman, "Computing Approximate Blocking Probabilities for A Class of All-Optical networks," IEEE Journal of Selected Areas in Communications, vol.14, no.5, Jun. 1996.

[3] B. Barry and P. Humblet, "Models of Blocking Probability in All-Optical Networks With and Without Wavelength Changers," IEEE Journal of Selected Areas in Communication, vol.14, no.5, Jun.1996.

[4] Z. Zhang and A. Acampora, "A Heuristic Wavelength Assignment Algorithm for Multihop WDM Networks With Wavelength Routing and Wavlength Reuse," IEEE/ACM Transaction on Networking, vol.3, no.3, Jun, 1995.

[5] S. Subramaniam, E. Harder and H. Choi, "Scheduling Multirate Sessions in Time Division Multiplexed Wavelength-Routing Networks," IEEE Journal of Selected Areas in Communications, vol.18, no.10, Oct. 2000.

[6] S. Ramesh, G. Rouskas and H. Perros, "Computing Blocking Probabilities in Multi-Class Wavelength Routing Networks," ACM Transactions on Modeling and Computer Simulation, 2000

[7] Y. Qin, K. Sivalingam and B. Li, "QoS for Virtual Private Networks (VPN) over WDM Networks," In Proceedings of Opticomm, 2000.

[8] D. Mitra, R. Gibbens, and B. Huang, "StateDependent Routing on Symmetric Loss Networks with Trunk Reservation - I," IEEE Transactions on Communications, vol. 41, no. 2, pp. 400-411, Feb. 1993.

[9] R. Braden et al.,"Integrated Services in the Internet Architecture: An Overview," RFC 1633, Jun. 1994.

[10] S. Blake et al.,"An Architecture for Differentiated Services," RFC 24751998.

[11] C. Dovrolis and P. Ramanathan, "Proportional Differentiated Services, Part II: Loss Rate Differentiation and Packet Dropping," In Proceedings of IWQoS, pp. 52-61, Jun. 2000.

[12] C. Dovrolis, D. Stiliadis, and P. Ramanathan, "Proportional Differentiated Services: Delay differentiation and Packet Scheduling," In Proceedings of ACM SIGCOMM'99, pp. 109-120, Aug. 1999. 
